\title{
INTERACTION BETWEEN THE SPIKE PROTEIN OF HUMAN CORONAVIRUS NL63 AND ITS CELLULAR RECEPTOR ACE2
}

\author{
Stefan Pöhlmann, Thomas Gramberg, Anja Wegele, Krzysztof Pyrc, \\ Lia van der Hoek, Ben Berkhout, and Heike Hofmann*
}

\section{INTRODUCTION}

Coronavirus $(\mathrm{CoV})$ infection of humans has so far not been associated with severe disease. However, the discovery of the severe acute respiratory syndrome (SARS) CoV revealed that highly pathogenic human $\mathrm{CoVs}(\mathrm{hCoVs})$ can evolve. As the characterization of new hCoVs is therefore an important task, we studied the cellular entry of hCoV-NL63, which was recently isolated from patients with lower respiratory tract illness. ${ }^{1}$

Entry of CoVs into target cells is determined by the major viral envelope glycoprotein termed "spike" (S), which provides the virions with their characteristic corona-like shape. ${ }^{2,3}$ The main function of the S-protein in CoV entry is the binding to a host cell receptor followed by fusion of viral and cellular membranes. ${ }^{4,5}$ The domains in $\mathrm{S}$ that are required for membrane fusion locate to the C-terminal half of the protein (S2 subunit). Receptor engagement is conferred by the N-terminal S1 subunit; consequently, the S-protein of a given CoV can determine its cell tropism. Although the S-proteins of animal and human CoVs exhibit the same functional organization, particularly the S1 subunits differ in amino acid sequence, resulting in interaction with specific cellular receptors. Within group I CoVs, hCoV-NL63 is phylogenetically highly linked to hCoV$229 \mathrm{E}$, and especially the S-proteins of both viruses share a high sequence homology. The S-protein of hCoV-229E is known to employ the human aminopeptidase N (hAPN, also called CD13, herein referred to as hAPN/CD13) for infection of target cells ${ }^{7}$; therefore, it was speculated that NL63 might use the same receptor for cellular entry. ${ }^{1}$

The S-protein is sufficient to mediate CoV entry into receptor-positive target cells and can be incorporated into heterologous viral particles. Thus, CoV S-proteins can be expressed in the envelope of lentiviral particles, and these pseudotyped viruses ("pseudotypes") proved to be a useful experimental system to analyze SARS-CoV-S

* Stefan Pöhlmann, Thomas Gramberg, Anja Wegele, Heike Hofmann, University of Erlangen-Nürnberg, Germany. Krzysztof Pyrc, Lia van der Hoek, Ben Berkhout, University of Amsterdam, The Netherlands. 
mediated cellular entry. ${ }^{8-11}$ For production of pseudotypes, envelope-defective lentiviral genomes encoding a reporter gene and a plasmid encoding a CoV S-protein are expressed in cells, which then secrete lentiviral particles harbouring the S-protein in their envelope. These particles infect susceptible cells in a CoV-S dependent manner, and entry efficiency can be quantified by determination of the reporter gene activity.

\section{IDENTIFICATION OF ANGIOTENSIN-CONVERTING ENZYME 2 (ACE2) AS A RECEPTOR FOR hCoV-NL63}

\subsection{The S-proteins of hCoV-NL63 and -229E Interact with Different Receptors}

Replication competent hCoV-NL63 has been cultured on tertiary monkey kidney cells, ${ }^{1,12}$ but permissive human cell lines had not been identified so far. Using lentiviral pseudotypes, we therefore analyzed a panel of human cell lines for susceptibility to hCoV-NL63 S-mediated entry (chapter 4.2). Interestingly, the cell tropism measured for NL63-S was congruent with that observed for SARS-CoV, ${ }^{8,10,11}$ but differed significantly from that measured for $229 \mathrm{E}-\mathrm{S}$, suggesting that both glycoproteins might interact with different receptors despite their high amino acid identity. Furthermore, we were able to show that hCoV-NL63 does not engage feline aminopeptidase N (fAPN) in contrast to all CoVs of class I investigated so far. ${ }^{13}$ However, cells expressing the SARS-CoV receptor protein ACE2 ${ }^{14,15}$ were susceptible to NL63-S driven infection. This was unexpected as NL63-S has no striking homology to either the whole S1 subunit of SARS-CoV or the already identified ACE2 interaction domain in SARS-CoV-S, ${ }^{16}$ suggesting that both proteins either form a common three-dimensional structure that allows ACE2 engagement in a similar fashion or that both S-proteins evolved different strategies to target ACE2. The interaction between NL63-S and ACE2 was specific, as the closely related ACE1 protein did not react with NL63-S, and on the other hand, ACE2 was not able to confer 229E-S-mediated infection, suggesting that ACE2 is not a functional equivalent of fAPN in class I CoV entry.

\subsection{Direct Interaction Between ACE2 and NL63-S}

In order to investigate whether NL63-S directly contacts ACE2, we employed a FACS-based binding assay. For this, the NL63-S1 region was fused to the constant chain of human immunoglobulin ( $\mathrm{IgG})$, expressed in $293 \mathrm{~T}$ cells and concentrated from the culture supernatant. Then, NL63-S-IgG was incubated with cells expressing either hAPN/CD13 or ACE2 followed by staining with specific antibodies directed against the respective receptor protein to quantify receptor expression levels and an anti-human Fc antibody to detect the bound IgG fusion protein. Hereby, we demonstrated that NL63-S in contrast to 229E-S does not react with hAPN/CD13, but like SARS-CoV-S directly binds to ACE2. Finally, we analyzed the NL63-S interaction with ACE2 by employing ACE1 or ACE2 specific polyclonal antisera. Only the ACE2 serum interfered with infection by NL63-S-bearing lentiviral pseudotypes and replication-competent hCoVNL63, thus confirming that ACE2 is a receptor for hCoV-NL63. 


\section{MAPPING OF THE ACE2-INTERACTION DOMAIN IN NL63-S}

\subsection{Role of the Unique Domain in NL63-S}

As mentioned above, the S-proteins of hCoV-229E and hCoV-NL63 share an overall amino acid identity of more than 50\%. However, hCoV-NL63 S harbors a 178 amino acid N-terminal extension that is not present in 229E-S or any other known protein and that is therefore designated "unique domain". In order to investigate if the unique region is involved in ACE2 binding, we first analyzed binding of the isolated domain to ACE2 expressing cells in a FACS based binding assay. However, the unique domain alone did not show any interaction with ACE2, indicating that it does not serve as an independent receptor binding domain. Because it is possible that the unique domain might confer ACE2-binding together with other sequences in the S1-domain of hCoV-NL63 and possibly hCoV-229E, we constructed a chimeric mutant comprising the N-terminal 178 amino acids of NL63-S fused to the S1 subunit of 229E. This mutant, however, showed no ACE2-interaction, but bound to hAPN/CD13 as efficient as the wildtype protein, indicating that the unique domain does not interfere with hAPN/CD13 recognition and does not allow binding to ACE2. When the unique domain was removed from NL63-S, the remaining protein still bound ACE2 and showed no affinity for hAPN/CD13, confirming that the unique region is dispensable for ACE2 binding. In summary, these observations indicate that amino acids in the highly conserved S1 regions of NL63- and 229E-S confer specificity for the interaction with ACE2 and hAPN/CD13, respectively.

\subsection{Analysis of hCoV-NL63-S1 Deletion Mutants and Chimeric hCoVNL63-229E-S Variants}

In order to map which region in the NL63 S1-protein is responsible for targeting ACE2, we analyzed a panel of N-terminal S1-deletion mutants. By this, we were able to narrow down the ACE2 interaction domain in NL63-S to amino acids 232 to 741 . In parallel, we constructed chimeric S1-proteins comprising defined regions of NL63-S fused to complementary domains within 229E-S and analyzed them for hAPN/CD13 and ACE2 interactions. We found that several sequence elements in the center and Cterminus of the proteins can impact receptor binding, suggesting that some of these mutations might interfere with the integrity of the possibly complex three-dimensional structure of the proteins and thus with their capacity to recognize receptors. Our observations are in agreement with a model suggesting that the central region in the hCoV229E-S and possibly NL63-S proteins might determine the correct folding or orientation of a C-terminal receptor binding domain, as has been suggested previously for hCoV-229E-S. ${ }^{17,18}$ Taken together, a detailed point mutagenesis of NL63-S1 will be required to identify residues with a critical function in ACE2 interaction. 


\section{DISCUSSION AND OUTLOOK}

We were able to show that the SARS-CoV receptor ACE2 is used by the recently identified hCoV-NL63 for entry into target cells. Simultaneously, the same observation was reported by Smith and colleagues (M.K. Smith, et al., chapter 4.13). The interaction of NL63-S with ACE2 was unexpected, as NL63-S and SARS-CoV-S share no significant amino acid homology. In contrast, NL63-S is highly related to the glycoprotein of hCoV-229E, which binds hAPN/CD13, and the hAPN/CD13 interaction domain is well conserved in NL63-S. The most striking difference between the S-proteins of hCoV-NL63 and -229E is a 178 amino acid extension that is exclusively present in NL63-S. This unique domain, however, is dispensable for ACE2-interaction; thus, amino acids in the highly conserved central portions and C-termini within the S-proteins of hCoV-229E and hCoV-NL63 determine the recognition of their respective receptors. Therefore, detailed point mutagenesis in combination with the determination of the threedimensional structure of both S-proteins is required to identify amino acids that mediate receptor binding. These data in turn will help to develop specific small molecule inhibitors against NL63-S-mediated infection.

\section{REFERENCES}

1. van der Hoek, L., et al., 2004, Identification of a new human coronavirus, Nat. Med. 10:368.

2. Holmes, K. V., 2001, The Coronaviridae, in: Fields Virology, D. Knipe, ed., Lippincott Wiliams \& Wilkins, Philadelphia, pp. 1187-1203.

3. Stadler, K., et al., 2003, SARS-beginning to understand a new virus, Nat. Rev. Microbiol. 1:209.

4. Gallagher, T. M., and Buchmeier, M. J., 2001, Coronavirus spike proteins in viral entry and pathogenesis, Virology 279:371.

5. Hofmann, H., and Pöhlmann, S., 2004, Cellular entry of the SARS coronavirus, Trends Microbiol. 12:466.

6. Kuo, L., et al., 2000, Retargeting of coronavirus by substitution of the spike glycoprotein ectodomain: crossing the host cell species barrier, J. Virol. 74:1393.

7. Yeager, C. L., et al., 1992, Human aminopeptidase $\mathrm{N}$ is a receptor for human coronavirus 229E, Nature 357:420.

8. Hofmann, H., et al., 2004, S-protein of severe acute respiratory syndrome-associated coronavirus mediates entry into hepatoma cell lines and is targeted by neutralizing antibodies in infected patients, J. Virol. 78:6134.

9. Moore, M. J., et al., 2004, Retroviruses pseudotyped with the severe acute respiratory syndrome coronavirus spike protein efficiently infect cells expressing angiotensin-converting enzyme 2, J. Virol. 78, 10628.

10. Simmons, G., et al., 2004, Characterization of severe acute respiratory syndrome-associated coronavirus (SARS-CoV) spike glycoprotein-mediated viral entry, Proc. Natl. Acad. Sci. USA 101:4240.

11. Yang, Z. Y., et al., 2004, pH-dependent entry of severe acute respiratory syndrome coronavirus is mediated by the spike glycoprotein and enhanced by dendritic cell transfer through DC-SIGN, J. Virol. 78:5642.

12. Fouchier, R. A., et al., 2004, A previously undescribed coronavirus associated with respiratory disease in humans, Proc. Natl. Acad. Sci. USA 101:6212.

13. Tresnan, D. B., and Holmes, K. V., 1998, Feline aminopeptidase $\mathrm{N}$ is a receptor for all group I coronaviruses, Adv. Exp. Med. Biol. 440:69.

14. Li, W., et al., 2003, Angiotensin-converting enzyme 2 is a functional receptor for the SARS coronavirus, Nature 426:450.

15. Wang, P., et al., 2004, Expression cloning of functional receptor used by SARS coronavirus, Biochem. Biophys. Res. Commun. 315:439.

16. Wong, S. K., et al., A 193-amino acid fragment of the SARS coronavirus S protein efficiently binds angiotensin-converting enzyme 2, 2004, J. Biol. Chem. 279:3197.

17. Bonavia, A., et al., 2003, Identification of a receptor-binding domain of the spike glycoprotein of human coronavirus HCoV-229E, J. Virol. 77:2530.

18. Breslin, J. J., et al., 2003, Human coronavirus 229E: receptor binding domain and neutralization by soluble receptor at 37 degrees, J. Virol. 77:4435. 